\title{
Hans-Joachim Giegel \\ Widersprüche zwischen gewerkschaftlicher Interessenvertretung und dem Alltagsbewußtsein von Industriearbeitern
}

Die Stellung der Gewerkschaften zwischen Lohnabhängigen und Kapital bringt es mit sich, daß gewerkschaftliche Politik die Interessen von Lohnabhängigen vertreten will, gleichzeitig aber zu den faktisch existierenden Interessen ihrer Mitglieder nach vielen Seiten hin in Widerspruch tritt. Aus der Sicht der Gewerkschaften liegt es nahe, den Grund dieses Widerspruches darin zu sehen, daß sie selber aufgrund der in ihr zusammenfließenden vielfältigen Erfahrungen und der von ihr vorgenommenen umfassenden Analysen eine so gründliche Einsicht in die durch ihre Politik berührten Zusammenhänge besitzen, wie sie das gewöhnliche Gewerkschaftsmitglied mit seinem bornierten Alltagsbewußtsein nicht entfernt zustandebringen kann. Dabei kann sich die Borniertheit dieses Bewußtseins sowohl dahin auswirken, daß die faktisch von den Mitgliedern geäußerten Interessen hinter den gewerkschaftlich vertretenen zurückbleiben, wie umgekehrt dahin, daß sie über diese hinausschießen. Daß die Linie, an denen das letztere Verhältnis in das erstere umschlägt, gewöhnlich zwischen Lohn- und Arbeitszeitinteressen auf der einen und Interessen an einer sinnvollen Tätigkeit, an der Erhaltung der Arbeitskraft, an verbesserten Reproduktionsbedingungen usw. auf der anderen Seite verläuft, wird als weiterer Beweis für die Borniertheit der Bewußtseins- und Interessenlage der Lohnabhängigen genommen.

Im folgenden geht es ausschließlich um das Gesundheitsinteresse von Lohnabhängigen. $\mathrm{Da}$ die Gewerkschaften gerade auf diesem Feld Schwierigkeiten haben, ihre Mitglieder hinter sich zu bekommen, wird von ihnen selber beklagt und häufig als Grund für ihr nur zögerndes Engagement in diesem Bereich angeführt. Es ist auch für gewerkschaftliche Funktionäre nicht leicht, den paradoxen Sachverhalt sich verständlich zu machen, daß die Sicherung der Gesundheit zwar im Interessse eines jeden Lohnabhängigen liegen müßte, jedoch eine mit diesem Ziel geführte Gewerkschaftspolitik oft auf unerwartete und hartnäckige Widerstände der Betroffenen stößt. Erklärt wird dieses Verhalten meist damit, daß bei den Lohnabhängigen das Lohninteresse eine absòlute Priorität besitze, die es keinem anderen Interesse gestatte, sich adäquat auszubilden. Es ist zu vermuten, daß dieses Erklärungsmuster selbst in den Fällen, die ihm noch am ehesten zu entsprechen scheinen, zu kurz greift. Sicher aber ist, daß es in anderen Fällen an der Realität glatt vorbeigeht. Am Beispiel des im folgenden analysierten Falls wollen wir demonstrieren, daß noch ganz andere als die gewöhnlich unterstellten Motivzusammenhänge das Interesse eines Lohnabhängigen an der Sicherung seiner Gesundheit blockieren können. Das von uns gewählte Beispiel scheint auf den ersten Blick sehr extreme Züge aufzuweisen und darum nur einen geringen Erklärungswert zu besitzen. Aber dieser Eindruck täuscht. Es ist zu vermuten und mehr als eine Vermutung erlaubt der erreichte Stand des Wissens hier nicht - daß erstens Fälle dieser Art, wenn auch nicht in der gleichen extremen Ausprägung, relativ häufig sind. Und daß zweitens selbst in den Fällen, die auf den ersten Blick ganz anderer Natur zu sein scheinen, sich oft die an unserem Beispielsfall entwickelten Motivzusammenhänge auffinden lassen. 
Wir müssen uns bei der Darstellung des Falls darauf beschränken, zu zeigen, wie aus einem bestimmten Motivzusammenhang Konsequenzen erwachsen, die den Anstrengungen einer gewerkschaftlichen Politik entgegenwirken. Welche Konsequenzen sich daraus für eine Gewerkschaftspolitik ergeben, muß in einem anderen Zusammenhang entwickelt werden.

(Anm. Das Interview, auf das sich die folgende Darstellung stützt, wurde im Rahmen eines von Joachim Heidorn und mir geleiteten Forschungsprojekts geführt. Um den Leser mit der Fallgeschichte vertrauter zu machen, wird auf das Transskript in seiner Originalform zurückgegriffen und werden möglichst viele - auch ähnlich ausfallende Aussagen - des Befragten zu einem Thema zitiert.)

\section{Eine Fallstudie zum Gesundheitsverbalten}

\section{Hinweise zur Berufsbiographie von Herrn $Z$.}

Herr Z. wurde 1933 in Rumänien geboren. Seine Eltern gehörten der Volksgruppe der Donauschwaben an, einer Minderheitengruppe deutscher 'Abstammung, die sehr darauf bedacht war, ihre eigene kulturelle Identität gegenüber der rumänischen Bevölkerung zu bewahren. Mit 16 Jahren, nach Abschluß der Schule, macht Herr $Z$. eine zweijährige Lehre als Schmied und Schlosser durch und zwar in einem Bergwerksbetrieb, in dem sein Vater als Bergmann arbeitet. In den darauffolgenden zweieinhalb Jahren absolviert er seinen Militärdienst. Danach ist er in seinem alten Betrieb als Schmied und Schlosser tätig. 1956 verläßt er mit einer Gruppe von 10 Leuten seinen Heimatort, geht nach Österreich und reist noch im selben Jahr von dort aus in die Bundesrepublik ein. Hier kann er durch Vermittlung des Arbeitsamtes nach kurzer Zeit in einem metallverarbeitenden Großbetrieb die Stelle eines Schmieds antreten. In diesem Betrieb ist er zum Zeitpunkt der Befragung seit 25 Jahren tätig und hat während dieser Zeit bis auf eine kurze Periode, in der er wegen Auftragsmangel innerbetrieblich versetzt wurde, seinen Arbeitsplatz nicht gewechselt. Nicht unerheblich verändert hat sich in dieser Zeit aber seine Arbeit und zwar wegen technologischer Umstellungen, die an seinem Arbeitsplatz vorgenommen wurden. So sind die offenen Feuerstellen durch Elektroöfen ersetzt worden, und zum Heben der schweren Eisenteile, das vorher mit Körperkraft erledigt werden mußte, werden jetzt Magnete eingesetzt. Diese Modernisierungen sind wie üblich von einer Erhöhung der von den Beschäftigten einzuliefernden Stückzahlen begleitet worden.

\section{2. "Daß ich diese schwere Arbeit mit 55 oder mit 60 Jabren bestimmt nicht mebr macben kann, das eine weiß ich."}

Im Laufe der Befragung kommt Herr $Z$. auf eine Fülle von Gesundheitsproblemen zu sprechen, von denen er betroffen ist: Bandscheibenschäden, Abnützung der Gelenke, Krampfadern, Bindegewebsschwäche in der Leiste (mit Neigung zu Leistenbruch), deutliches Nachlassen der Kräfte, Schwerhörigkeit und Neigung zu länger anhaltenden Katarrhen mit Schnupfen, Husten und Kopfweh. Herrn Z.' Bericht von diesen Krankheiten ist ohne Beschönigung: er erzählt von zum Teil heftigen Schmerzen, die sie ausgelöst haben, von 
den Einbrüchen in sein alltägliches Leben, die sie zur Folge hatten, und von der Möglichkeit der Wiederkehr der Symptome oder sogar der Ausweitung von Gesundheitsproblemen über das bisher erfahrene Maß hinaus. Bemerkenswert realistisch ist auch seine Einschätzung der weiteren Entwicklung seines Gesundheitszustandes: er erwartet, daß sein Gesundheitszustand sich in nicht all zu ferner Zukunft - er rechnet mit 5 bis 7 Jahren - so verschlechrert haben wird, daß er seine jetzige Arbeit aufgeben muß. Er rechnet darüber hinaus damit, daß dieser frühzeitige Gesundheitsverschleiß sich ungünstig auf seine Lebenserwartung auswirken wird.

Nicht nur wegen dieser realistischen Sicht der an seinem Körper sich vollziehenden Gesundheitszerstörung, sondern auch wegen der von ihm vorgenommenen Zuschreibung der Ursachen, die für diese Zerstörung verantworlich sind, wäre zu erwarten, daß Herr Z. sein Schicksal nicht einfach hinnimmt. Zwar führt er einige seiner Krankheiten auf Vererbung oder frühe traumatische Einwirkungen zurück, denen sein Körper ausgesetzr war, aber gerade die schweren Krankheitssymptome, insbesondere diejenigen, die ihn zwingen werden, seine Arbeit aufzugeben, sieht er durch diese Arbeit verursacht.

Befragter (B): ... Ich hab's jetzt schon ein wenig mit den. Bandscheiben, durch das eintönige Arbeiten, ne.

Interviewer (I): Ja, mmh, Mmh

$B$ : Die Bandscheiben, das ist mit der Zeit durch die schwere Arbeit, ist das schon bei mir schon a weng abgenützt, aber ich geb's trotzdem nicht, ich geb nicht locker, ne...

I: Was hat der Arzt denn so über die Ursache gesagt?

$B$ : Ja, das ist des überanstrengend, laufender schwerer Arbeit. Und das Eintönige, ne. Beispiel wie bei mir die Bandscheiben (...), da ich immer wieder auf rechts auf links rüberziehe, es ist immer die, die eine Arbeit, ne, ne, des wechselt ja nicht, daß ich mal do, mal do rüber ... und des, sagen mir, mit de laufende Jahre, die wir schon darin sind, des haben die alle, ne...

$B: \mathrm{Ja}, \mathrm{Ab}-$, A bnützung sind schon mal. Ich hab damals die Abnützung gehabt sehr, do in des Gelenk, des haben se halt bei uns alle ne, durch des schwere Heben, ne...

$B$ : Nee. Das ist das einzige Mal. Den Abnützungen, des hat bei uns a jeder, wenn einer schon mal so lange drin, mit de schwere Brocken umgeht, ne...

$B$....daß ich diese schwere Arbeit, sagen mir mit, mit $55 \mathrm{Jahr}$, mit $60 \mathrm{Jahr}$ bestimmt nicht machen kann, des, des eine weiß ich, das weiß ich. ... Aber daß ich dann irgendwie e leichtere Arbeit kriege, ne. Des, des, wenn ich des noch fünf Jahre mach, dann glaub ich, wird's, wird's so weit sein, ne, ne, I: Wenn Sie mal so die Arbeitssituation insgesamt betrachten, meinen Sie, äh, ... fünf Jahre

$B$ : Ja, fünf Jahre will ich schon noch machen, ne...

I: Ja.

B: I mein, dann bin ich 53, 54, ne. Und dann, ob's mir dann noch so geht, wenn's geht, will ich schon bleiben noch weiter, ne. Aber ich sag, ich will hat, will halt arbeiten. Und wenn's nicht geht, na dann, kaputt machen, will ich mich auch wegen dem nicht lassen, ne. Mei, man muß halt, muß halt dann mit der leichteren Arbeit auch zufrieden sein. Ma's is, wenn ich halt in der Kontrolle bin, hab ich dann nicht den, den großen Akkord wie was ich in der großen Maschine auch, ne. Aber dann muß des auch reichen, wenn die anderen dene reicht's ja auch aus.

In seiner Ursachenzuschreibung wird er offensichtlich durch die Erfahrung gestützt, daß gerade die schweren Krankheiten nicht nur ihn getroffen haben, sondern ein gemeinsames Schicksal aller Kollegen sind, die in seiner Abteilung arbeiten.

Wie verhält sich Herr $Z$. gegenüber den von ihm klar realisierten und auf seine berufliche Tätigkeit zurückgeführten Störungen seiner Gesundheit? 


\section{3. "Ich sag immer, so alt möcht ich gar nicht werden."}

Nach Herrn Z.s Einstellung zu seinen Gesundheitsproblemen brauchen wir nicht lange zu suchen. Sie ist im ersten Satz, in dem er auf seine Gesundheit zu sprechen kommt, ausgedrückt - in einem Satz, der nach den soeben beschriebenen Erfahrungen und Überlegungen von Herrn $Z$. verwirtend und rätselhaft erscheinen muß:

I: Gut, jetzt haben wir einmal über die Berufslaufbahn getedet, äh, jetzt kommen wir mal auf die Gesundheit. Und da hab ich am Anfang hab ich hier ...

$B$ : Ja, da könnt ich schon was sagen

I: ... so ne Stufenleiter. Und da wollt ich mal folgendes fragen. Wenn Sie mal hier so sehen von 0 bis 10. 10 bedeutet kerngesund, 0 bedeutet schwere Krankheiten. Wo würden Sie sich denn da und Ihre Gesundheit, wo würden Sie die da einordnen?

$B: \mathrm{Na}$, ich war bis jetzt noch kerngesund gewesen, bis auf en Leistenbruch ne.

I: Ja, wann war das?

$B$ : Der Leistenbruch, der wat voriges Jahr gewesen

I: Ja ja wo würden Sie sich denn jetzt da so einordnen, insgesamt?

$B$ : Als kerngesund.

I: Als kerngesund, ganz oben, also hier bei 10, ja

$B$ : Ganz oben ganz genau

I: Ah ja. Mmh

$B$ : Na ja, bis jetzt war ich noch nicht krank gewesen, na so selber a Grippe hab ich ma gehabt. Na ja, da hab ich da war ich aber schon gestanden fast auf 40 Fieber. Da ham's gesagt, jetzt wird die höchste Zeit, wenn's d'heim gehst.

I: Mmh wann war denn das?

$B:$ Das war vor fünf Jahren gewesen

I: Danach haben Sie ...

$B$ : No, noch nichts gehabt ...

$B$ : Aber so richtige Krankheiten hab ich noch nicht gehabt.

I: Gar nicht gehabt

$B$ : Einmal a Grippe gehabt, das wat alles.

Auf die Eingangsfrage nach seinem gegenwärtigen Gesundheitszustand bringt Herr Z nicht nur die meisten seiner Gesundheitsprobleme nicht zur Sprache, sondern bezeichnet sich explizit als kerngesund. Nun ist es nicht so, als würde Herr Z. seine Krankheiten nicht bemerken oder sich nicht mehr an sie erinnern. Bei Gelegenheit kommt er in seinen Erzählungen auf sie zu sprechen und zwar so, daß man annehmen muß, daß sie ihm auch am Anfang des Interviews präsent waren. Vieles von dem, was Herr Z. später im Interview äuBert, spricht für eine ganz andere Erklärung der hier von Herrn $Z$. vorgenommenen Einschätzung seiner Gesundheit: für Herrn Z. stellen Gesundheitsprobleme, wie er sie erfahren hat, auch die recht gravierenden Probleme, die im Kern gesunde Natur des Körpers nicht in Frage, und zwar deshalb nicht, weil er die für ihn zentrale Lebenstätigkeit, nämlich seine Arbeit, unbehindert ausführen kann. In diesem Sinn kennt er keine Gesundheitsprobleme, ist er kerngesund. Es handelt sich hier also nicht um eine bloß terminologische Verschiebung, sondern Herr Z . will zum Ausdruck bringen, daß er mit dem Zustand des 'Kerngesundseins' zufrieden ist, weil er damit über alles für ihn Notwendige verfügt, und daß er deshalb auf alles Übrige wie auf einen überflüssigen Luxus verzichten kann.

Aber dies ist nur die erste Stufe des Verzichts, der von Herrn Z. ausgesprochen wird. Wir wissen schon, daß Herr $Z$. für die Zukunft nicht einmal eine Bewahrung des jetzigen, 
schon prekären Gesundheitszustandes erwartet. Auffallend ist, daß er sich in keiner Weise über das von ihm für die Zukunft erwartete Schicksal beklagt und schon gar nicht sich gegen dieses Schicksal auflehnt. Es wäre nicht einmal zutreffend, wenn man sagte, er habe sich in sein Schicksal hineingefunden, denn das würde ja noch einen anfänglich bestehenden Gegensatz zu diesem beinhalten. Die in Zukunft zu befürchtenden Gesundheitsprobleme werden ganz unsentimental als Teil eines Lebenszusammenhangs, den man als ganzen akzeptiert, eingeführt, sie gehören zu diesem Leben in dem Sinn, daß etwas anderes gat nicht erwartet werden könnte.

Aus diesem Kontext heraus wird erst der spezifische Sinn des Satzes »Das hat bei uns jeder« interpretierbar. Entgegen den Vorurteilen, in denen sich eine auf bloße Spekulation gegründete Analyse des Arbeiterbewußtseins ergeht, kann die Vergegenwärtigung eines gemeinsamen Schicksals der Produzenten nicht nur die Funktion haben, sich im Kampf gegen den identischen Urheber dieses Schicksals zu verbinden, sondern kann umgekehrt auch zum Verzicht und zur Ergebenheit in das eigene Schicksal motivieren, wenn nämlich aus dieser Erkenntnis nur die tröstliche Gewißheit gezogen wird, daß man mit seinem traurigen Los nicht schlechter als andere dasteht.

Mit der Hinnahme zukünftiger Gesundheitszerstörungen ist Herr $Z$. auch bereit, die problematischen Folgen, die mit diesen Gesundheitszerstörungen verbunden sind, $z$. B. den Wechsel zu einer Arbeit, bei der er nicht mehr so viel verdient wie jetzt, zu akzeptieren. Besonders aufschlußreich ist das letzte Glied der Kette von Verzichtserklärungen, nicht nur weil damit noch einmal die Konsequenz vorgeführt wird, mit der Herr $Z$. seinen Verzicht, was seine Gesundheit anbelangt, vertritt, sondern vor allem wegen eines an dieser Stelle ausgeprochenen Hinweises auf die Gründe, die Herrn Z. zu diesem Verzicht bewegen,

I: Glauben Sie, daß die Belastungen, unter denen Sie auch jetzt noch stehen so in der Arbeit, daß die irgendwie im Alter sich mal auswirken könnten?

$B$ : Ja noch die anderen noch, wie ich schon gehört hab, ma die haben's immer schon gesagt: Na ja, wenn's de schwer arbeitest, das wirst schon noch merken später, ne, aber ma ... wie was ich halt von dene gehört hab, ne, und sei halt mit ... mach, such dir e leichtere Arbeit, ne, und mei ich hab mich da nit einflößen lassen. Ich hab gesagt, na ja das werd ich schon merken dann. Du wirst auch merken, wenn's zu spät ist, ne, sagen se halt, ne. Aber selber könnt ich nicht sagen. Kann sein, daß ich's merken werden, freilich (...) das sieht man ja bei die anderen, die ma, die was sagen ma mal ewig so wie bei mir der Groß... von meiner Frau der Großvater do, ne, der der ist 86, 6.., 89 Jahre alt, ja der Mo, der macht doch seine, seine Spaziertouren und alles, da sagt man halt, na ja da er immer gesagt hat, er hat sich noch nie in seinem Leben geplagt, ne. Ich sag immer, so alt möcht ich gar nicht werden, ne. Das sind halt, sag mal, so Meinungen, ne.

Herr Z. weiß: man könnte seine Gesundheit bewahren, wenn man nicht arbeiten und sich bei der Arbeit nicht »abplagen« würde. Aber damit ist nicht die »Meinung«, d. h. das Interesse von Herrn Z. getroffen. Er ist bereit zu arbeiten und dabei seine Gesundheit aufs Spiel zu setzen. Die Vermutung liegt nahe, daß im Bereich der Arbeit auch das zu finden ist, was Herrn Z. zu seinem Verzicht motiviert und diesen Verzicht erklärt.

\section{4. "Ich will bloß nur schaffe, mur schaffen"}

Herr Z. läßt in seinen zahlreichen Äußerungen zu seiner beruflichen Tätigkeit keinen Zweifel daran, daß ihm diese Arbeit nicht nur Spaß macht, sondern er sich uneinge- 
schränkt mit ihr identifiziert. Das ist einerseits im konkreten Charakter, nämlich der Naturnähe der Schmiedearbeit begründet. Der unmittelbare Umgang mit den 'Naturelementen' und der Prozeß der Zähmung dieser Elemente, der diese zwingt, zu dem bezweckten Resultat zusammenzuwirken, scheint auf Herrn Z. eine Faszination auszuüben.: "Mir hat das immer Spaß gemacht, mit dem Feuer umzugehen. ....

Wichtiger noch ist, daß eine solche Arbeit eine Herausforderung darstellt, die nur zu bewältigen ist, wenn der Arbeiter über eine Reihe von Eigenschaften verfügt: die Kenntnis der richtigen Vorgehensweise, das Gespür für die Eigentümlichkeiten des Materials, die Fähigkeit mit den unvorhersehbaren Tücken des Naturstoffes fertig zu werden, die körperliche Kraft, mit der die dem Material eigene Gewichtslast bewältigt werden muß, die Robustheit, ohne die im Arbeitsvollzug auftretenden Belastungen nicht ertragen werden können, schließlich die Hartnäckigkeit und Unermüdlichkeit, die zur kontinuierlichen Aufrechterhaltung des Arbeitsprozesses und damit zur Bewältigung der gesamten Masse der anfallenden Arbeitsaufgaben notwendig sind. Von der Arbeit erfüllt $z u$ sein, heißt für Herrn Z., ausgestattet mit allen diesen Eigenschaften sich den Herausforderungen des Arbeitsprozesses zu stellen und durch Bewältigung dieser Herausforderungen sich als guter Arbeiter zu beweisen.

$B:$... Jetzt hab wie ich dann dahin gekommen, ma die haben mich gut aufgenommen, also wenn ihr wollt, könnt ihr arbeiten ne, ma ich war 24 Jahre alt, und bin ich halt, hab ich halt gesagt, na ja, wo man halt das meiste verdienen kann, weil ich immer weng ehrgeizig war gewesen, ma das bin ich in der Arbeit drinnen genauso. Ich will bloß nur schaffen, nur schaffen.

$B:$... ich hab, hab mich gut eingefunden, sagen wir, mit die anderen Arbeitskollegen, mich haben sie gut aufgenommen, ich hab mit meinem 'Meister auch e gut's Verhältnis gehabt. Zu mir hat gesagt, für mich war es momentan schon schwer gewesen, weil ich trotzdem vieles nicht verstanden hab.

I: Ah ja

$B: \mathrm{Ne}$, ne, aber ich hab dann das schon gesehen, also wies de ganze Schmiederei sich vorgeht, das ist ja keine Freischmiede, das ist ja eine Formschmiede, ne, ne. Und hab ich gedacht hab, ne, es bleibt mir nichts anderes übrig. Entweder oder. Entweder du schaffst oder

$B ;$... also du mußt es schaffen, ne. Ne und da ... mei es waren schon schwere Tage gewesen, da hab ich auch mit mir geschimpft, hab, ne Herr Gott Dunnerwetter nei, ne und so und so. Aber das hat sich dann wieder gelegt.

B: ... gut ist mal, es ist nicht jeder Tag ein Fangtag, sag ich immer wieder. Der eine Tag ist halt immer wieder e weng schlechter, der andere Tag nun ja, dann wird man's wieder neingeholt, ne, ne.

$B$ : Und dann muß des hinhauen halt, wenn's halt e mal nit hinhaut e mal en Tag, Gott, ich sag immer wieder, gut es hat halt nicht so sein sollen, dafür müssen wir halt morgen mal energischer durchhauen.

$B$ : Wenn jetzt Beispiel die Decke hab gehabt, da hab ich manchmal drei Wochen dran gearbeitet, das geht ein manchmal bis daher $(. .$.$) was also was jetzt höchste Zeit wenn wird was anderes werden. Ob-$ wohl man verdient da sein Geld genauso damit, ne, ist auch a weng leichter, aber durch das Eintönige, ich will, ich hätt gern, daß, das sagt a jeder bei uns drinnen, a weng schon Wechsel, also das ist schon besser wie wie das jetzt ewig die Motoren haben. Und dann macht bloß noch die, die Gummischläuche, die Wasserschläuchle drauf, ne dann läuft das am Band weiter, der macht bloß noch des drauf, der mach des, ne. Der is dann ne so, do weiß man genau, noch so und soviel Maschinen machen die bring ich zusammen. Und ich will mehr, sagen wir mal, mehr so a weng kämpfen dafür, ne, ne. Das, das e mal ist okay, aber morgen muß wieder gehen, und macht ein Stück und am nächsten Tag will man sagen, Mensch wenigstens eir Stück mehr, ist schon ..., aber das ist halt alles so, so Spinnereien. 
Was für den unmittelbaren Arbeitsprozeß gilt, das gilt auch für die gesamte Berufslaufbahn: nur dadurch kann man Befriedigung in seinem Berufsleben erreichen, daß man sich den Anforderungen, die an einen gerichtet werden, stellt und schrittweise durch Ausräumen der Schwierigkeiten in die Position eines guten und anerkannten Arbeiters vorrückt.

$B$ : Jetzt verstehn ich's jetzt verstehn ich's. Ja. Ich hab immer wieder gesagt, der Gott hat mir den Tag geschaffen, daß ich nicht en einfach rumtrödeln würde, sondern man soll den Tag auch erfüllen, und ich freu mich jedes Mal, wenn ich sag, gut des is, ne, das Material hast du geschafft, das war deine Verantwortung, hob mich auch damit sehr viel mit dem Meister mich in Verbindung gesetzt, mit dem Herrn K., der gesagt hat, na ja also, es ist immer doch besser, sag ich, wenn ich sag, wie wenn ich bloß des ... ich will damit nicht die anderen Leute sag mal runterschauen, des absolut nicht, aber wenn ich sag, gut jetzt die Verantwortung hast du ja gehabt, daß des, des, des hergezeigt hast, also des, des rausgeschmiedet hast, ne ... und mei, so war ich schon immer gewesen.

I: Ah, ja

$B$ : War ich schon immer gewesen. Ja. Denn ich hab drüben in Rumänien schon in mein zweiten Lehrjahr schon selber schon auf Akkord gearbeitet, obwohl mein Lehrjahr war, ne, hab wohl nicht gekriegt, aber ... ich war schon immer so ehrgeizig gewesen.

Charakteristisch für Herrn $Z$. ist aber nicht nur, daß er das Motiv, ein guter Arbeiter zu sein, überhaupt besitzt. Das Spezifische, was durch das ganze Gespräch hindurch und in vielen Äußerungen sich geradezu aufdrängt, ist vielmehr.die Stärke, mit der dieses Motiv im Leben von Herrn Z. wirksam ist. Wenn die Lohnarbeit wegen ihrer Ausdehnung und den mit ihr verbundenen Belastungen auch dann das Leben eines Arbeiters beherrscht, wenn dieser sich nicht mit seiner Arbeit identifiziert, so treibt das Interesse von Herrn Z., ein guter Arbeiter zu sein, die Vereinnahmung des Lebens durch die Arbeit noch ein beträchtliches Stück weiter.

$B$ : Da sag ich, ich will nicht einfach, na ja gut jetzt, mal zum Beispiel wie die Helfer sind, da sind sehr viel verschiedene, ne, die sagen einfach, hauptsächlich, ich hab mein Tag rum. Ob ich jetzt meine Minuten verdient hab, der Kapo, der muß ja einliefern, ne, ne. Und das hat natürlich mir absolut noch nie gepaßt, ne. Ne, ich geh ja, steh schon um vier Uhr auf. Um sechse fäng ich an, um vier steh ich schon auf, trinke e mei Kaffee schnell oder irgend Kaffee halt, ja und dann lauf ich nauf und dann bin ich schon um, um Viertel sechs [ = 51/4] bin ich schon an meinem Arbeitsplatz, ne, und dann tu ich schon mei Material nei tun und mit mir noch ein Arbeitskollege was auch was auch noch in meiner Gruppe ist, der ist auch immer dort, ne, der tut seine, der tut schon die Maschine schon abschmieren jeden Tag. Und die anderen, die kommen halt genau entweder fünf Minuten nach sechs oder eine Minute vor sechs, ne. Ma sowas ärgett mich schon, ne

I: Gibt's denn wenn Sie so aus dem Betrieb kommen, gibt's denn so Dinge, die noch so in Ihnen nachwirken?

$B$ : Na ja, das mal was, wenn's momentan den Tag nicht richtig geloffen is, daß man abends sich do e wegen so lad ist oder irgendwas da ich muß sagen: Mensch mei du, warum ist das nit gegangen, daß man nachdenkt, des des ist bei mir öfters.

I: Das ist schon mal, ja.

B:Ja. Und wenn ich weiß, daß e ganz gefährliche Arbeit ist und denk, n' hoffentlich geht der 'Tag rum heut wieder, ne, daß, daß ich das herschaffen tu, do denk ich sehr viel da nach hinterher. Da denk ich schon da nach. Da, da kann ich manchmal abends, träume ich sogar noch davon, ne. Da sind momen$\tan$ vielleicht die Nerven e weng, daß man' sagt, na ja, das ist so gangen und und hoffentlich haut's hin, wenn, wenn' doch nicht das richtige Material nicht so ist, das reißt, warum reißt das, daß man do nachdenkt. Da denk ich schon nach, do, do werd ich e weng schon nervös dann irgendwie aber, da (was hat nit?) also richtig nervös, ich kann nicht arbeiten oder was, ne. Do, do denk ich schon nach sehr viel. 
I: Wenn Sie so drei Wochen Urlaub gemacht haben, haben Sie dann das Gefühl, jetzt reicht's wieder, jetzt muß ich mal wieder an die Arbeit ran? Oder könnten Sie noch weiter...

$B$ : Ich kann vollständig ausschalten. Aber dann die letzte Woche, die letzten drei vier Tag, hab ich bloß noch die Arbeit im Kopf dann.

$B$ : Beispiele, wenn ich weiß, daß ich am Montag anfangen muß und am Freitag kommt's mir's dann, Freitag und Samstag, Sonntag, ne, hab ich schon keine Ruh mehr, dann entweder ruf ich mit dem 'Telefon mei Kollege an: Na was waren jetzt dadrin, was, was gibt's Neues, ne, läuft die Wo (?), wie wie seit ihr mit eurem Geld hinkommen, langen eine Minuten...

Es ergibt sich aus dem Gesagten, daß der enorme Arbeitseinsatz von Herrn Z. gänzlich mißverstanden wäre, würde er einfach als Resultat von Lohnanreizen oder gar als Folge eines äußerlichen, vom Betrieb auferlegten Zwanges begriffen. Es ist ein von Herrn Z. selbst ausgebildetes Interesse, ein sein ganzes Leben beherrschendes Ideal vom guten Arbeiter, das ihn zu seiner rastlosen Arbeitstätigkeit treibt. Zwar hilft er mit der extensiven Verausgabung seiner Arbeitskraft, den Betriebszweck zu fördern, aber diese seine Einordnung in den Betriebt erfolgt nicht durch Verleugnung der eigenen Interesen, sondern gerade durch konsequente Realisierung seines zentralen Interesses, ein guter Arbeiter zu sein. Herr Z . erfährt, daß er sich nicht eigens den Interessen der Firma unterordnen muß, da seine Auffassung von Arbeit und vom guten Arbeiter, die der sich gebildet hat, sich lückenlos mit dem deckt, was die Firma zu ihrem eigenen Erfolg benötigt. Und dieses Bild eines harmonischen Verhältnisses von Arbeiter- und Betriebsinteresse kann gerade dadurch überzeugen, daß Herr $Z$. die von ihm ausgeführte Arbeit ohne soziale Bestimmung denkt, als eine wesentlich durch den Naturstoff selbstbestimmte Tätigkeitsform, wodurch gerade die sozial vermittelten Momente, nämlich die konkrete Ausformung dieser Tätigkeit, der Umfang der Arbeitsaufgaben, die spezifische Gestaltung der Arbeitsbedingungen, die Zweckbestimmung der Arbeit, der Interessenzusammenhang, dem die Arbeit unterworfen ist, für ihn ganz in den Hintergrund treten.

Wenn aber in der beschriebenen Weise der eigene Zweck und der Betriebszweck zusammenfallen, so ist es selbstverständlich, daß man sich nicht nur mit den betrieblichen Instanzen »in Verbindung setzt« (vgl. o. S. 11) und »ein gutes Verhältnis zu ihnen hat« (S. 10), sondern, daß man sich auch den Aufforderungen der Firma zu besonderen Leistungen nicht verschließt. Ob es darum geht, Überstunden zu machen, vorübergehend den Arbeitsplatz zu wechseln, oder eine Schicht zu übernehmen, der Meister kann stets mit Herrn $Z$. rechnen: »Ma ich werd's immer machen.«

Für Herrn $Z$. ist es entscheidend, daß die Firma ihm die Möglichkeit bietet, das von ihm angestrebte Ideal eines guten Arbeiters zu realisieren und dafür im Betrieb Anerkennung zu gewinnen, Anerkennung sowohl für die Qualität seiner Arbeit wie den unermüdlichen Einsatz, mit dem er seine Arbeit betreibt. Welchen Wert Herr Z. auf diese Anerkennung legt, zeigt sich im Interview an den vielen Verweisen auf betriebliche Instanzen, die, weil er nie zu anderweitigen Vermutungen Anlaß gegeben hat, »wissen« und »behaupten können«, daß mit seinem Arbeitseinsatz unbedingt gerechnet werden kann.

$B: \mathrm{Ne}$, ma da könnens mei Meister fragen und auch der vorhergehènde Chef, der is schon pensioniert und der heutige ist der Herr Sch., ne, i ma aber, für mich sagen mal, also Eigenlob stinkt heißt's, aber ich hab das noch nie getan, daß geheißen hat, na ja hoffentlich vielleicht kummt der heut oder irgendwas, ich war jeden Tag àn meiner Arbeit gewesen, ne.

Sicher kann Herr Z. sich ausrechnen, daß die betriebliche Anerkennung ihm auch bestimmte Vorteile oder Sicherheiten eintragen kann, aber das ist nicht entscheidend. Diese 
Anerkennung ist für Herrn Z. ein wesentliches Mittel der Identitätssicherung und hat damit für ihn mehr als einen nur instrumentellen Wert.

Weil das Leben von Herrn Z. um sein ständiges Bemühen kreist, die Leistungen, die einen guten Arbeiter ausmachen, zu erbringen, wird die Firma, die ihm sowohl die Möglichkeit gibt, sich in diesem Sinn zu bewähren, wie auch die Anerkennung vermittelt, durch die Herr Z. sich bestätigt sieht, in einem besonderen Sinn zum Lebensraum:

I: Wenn Sie jetzt nochmal so den gesamten betrieblichen Alltag jemand erklären würden, vielleicht einem Außenstehenden erklären würden, womit würden Sie den Betrieb am ehesten vergleichen? Ich sag mal mit einer Familie, mit einer Fußballmannschaft ...

$B$ : lch sag, das ist meine zweite Heimat, sag ich, (lacht). Mein zwei-, mein zweites W ohnzimmer also, ne, mein zweites Zimmer halt, ne. So würd ich das vergleichen.

I: Mit en zweiten?

$B$ : Mit en zweiten Wohnung, halt.

I: Mit ner zweiten Wohnung.

B: Man, man sitzt halt, ich sitz ja fast schon drin auch, ne, so würd ich's, so würd ich's (...) hinstellen. $I:$ Würden Sie denn auch sagen, das ist für Sie auch so wie ein Heim?

$B$ : Na ja, mei wenn, mei ich komm jetzt von daheim, geh da weg, jetzt weil ich, daß ich hineingeh wie (?) in ne Halle, ne, ne und mei da, da kenn ich ja jetzt Ecken und Winkel, ich weiß alles, ne, ma durch die langen Jahre durch, ne. Da fühl ich mich halt wie daheim in der Wohnung halt, ne, bloß, das halt nicht so ausschaut (lacht), das ist der eine Unterschied.

Das von uns dargestelite Verhältnis zur Arbeit, das das Leben von Herrn Z. bestimmt, spiegelt sich auch in seinem Verhältnis zu seinen Kollegen wider. Trotz seiner wiederholt geäu ßerten Meinung, bescheiden sein zu wollen, und trotz solidarischer Gefühle, die er gegenüber Kollegen verspürt, kann er von seinem Standpunkt aus nicht anders, als hinsichtlich vieler Kollegen ein tiefes Unbehagen zu empfinden, wenn er sich ihre Einstellung zu Arbeit und zum Betrieb vorführt. Daß Kollegen nicht mit dem gleichen Eifer und mit dem gleichen Einsatz an ihre Arbeit gehen wie er selber, hat ihm »absolut noch nie gepaßt $\ll$.

\section{5. "Das war alles schon soweit gewesen, daß es nicht mehr gegangen ist."}

W ir haben das zentrale Lebensinteresse von Herrn Z. beschrieben, nämlich sein Interesse, ein guter Arbeiter zu sein, und wollen nun verfolgen, wie dieses Interesse sich auf sein Verhältnis zu Gesundheit und Krankheit auswirkt. Das letzte Resultat des hier bestehenden Wirkungszusammenhangs haben wir schon kennengelernt: den weitgehenden Verzicht auf Gesundheit, den Herr Z. sich zu eigen gemacht hat. Die beiden wichtigsten Zwischenglieder dieses Wirkungszusammenhangs sind einerseits das Verhältnis, das Herr Z. zu den seine Gesundheit gefährdenden Arbeitsbedingungen ausgebildet hat, (5.1) und andererseits sein Gesundheits- und Krankheitsverhalten, durch das seiner Gesundheit zusätzliche Gefahren erwachsen (5.2).

\subsection{Arbeiten unter gesundheitsgeführdenden Bedingungen}

Wenn Herr Z. seine Arbeit als ein Ringen mit dem Naturstoff erfährt, dann sind von dieser Arbeit die belastenden Arbeitsbedingungen nicht wegzudenken. Zur Schmiedearbeit ge- 
hören gleichsam von Natur aus die Notwendigkeit, "mit schweren Brocken rumzuhauen«, Hitze und Lärm, schlechte Luft usw. Nach Ansicht von Herr Z. muß deshalb auch, wer die Schmiedearbeit zu seinem Beruf macht, akzeptieren, daß er unter Bedingungen arbeitet, die eine schwere Belastung darstellen und auf längere Sicht zu Gesundheitsproblemen führen.

I: Wie würden Sie das ankreuzen?

$B$ : Na also die Hitze will ich, ich, ich mein, aber des, des nur, wenn's außen so heiß ist, wenn's schwül ist, ne wenn nit, des hat jetzt mit dem Matetial nichts zu tun, mit dem Material nichts zu tun, do die Hitze.

I: Mh das können Sie ganz gut aushalten die Hitze vom Material her?

$B:$ Ja des muß ich, des ist ja der Beruf, ne.

I: Schwere Lasten?

$B: \mathrm{Na}$, das ist der Beruf, da will ich gar nichts sagen.

B: Unfallgefahr? Na ja .. na ja wenn's passiert, ist's passiert. Das stört mich auch nicht.

Insbesondere für Herrn Z. können belastende Arbeitsbedingungen und die aus diesen resultierenden Gesundheitsprobleme nichts Fremdes sein, gegen das er sich wehren müßte, weil er gerade durch Ertragen und Aushalten dieser Arbeitsbedingungen den für seine Identität unverzichtbaren Beweis führen kann, ein guter Arbeiter zu sein. Von dieser Seite her betrachtet dürfte, um es überspitzt zu sagen, die Schmiedearbeit gar nicht wesentlich anders aussehen als so, wie Herr Z. sie erlebt. Dementsprechend beschäftigt Herrn Z. auch kaum der Gedanke, ob nicht Veränderungen der Arbeitsbedingungen versäumt oder verhindert worden sind, die zu einer entscheidenden Verbesserung seiner Lage hätte führen können. Und erst recht nicht würde er seine Firma wegen eines solchen Versäumnisses anklagen.

Aber schon dadurch, daß Herr Z. seine Arbeit zunächst weitgehend ohne soziale Vermittlung auffaßt, fehlt ein Ansatzpunkt dafür, um eine Differenz zwischen seinen Interessen und den durch die Firma hergestellten Arbeitsbedingungen festzustellen. Zu dieser Auffassung gehört gerade, daß der Maßstab der Schmiedearbeit aus ihrer unentwickelten, historisch überholten und gleichsam durch den Naturprozeß diktierten Form her gewonnen wird. Es erscheint Herrn Z. so, als würde sich das Wirken der Firma zunächst nur darauf beschränken, einen unabhängig von ihr strukturierten Arbeitsprozeß einzurichten. An diesem Maßstab gemessen aber müssen alle Eingriffe der Firma (oder auch der Gewerkschaften oder des Staates) in die Arbeitsbedingungen als Verbesserungen aufgefaßt werden, die als weiterer Beweis dafür gelten können, daß es ein den Interessen der Beschäftigten entgegenstehendes Interesse der Firma nicht gibt.

Die oft von Herrn Z. vorgetragenen Maximen 'kämpfęn' und 'ehrgeizig sein’ können aus dieser Sicht nur den Inhalt haben, sich unter vorgegebenen Bedingungen zu bewähren und gerade darum sich über die notwendige Versagung von anderen Interessen, auch des Gesundheitsinteresses, nicht zu beklagen, besser noch: diese Versagung gar nicht zur Kenntnis zu nehmen. Das Gesundheitsinteresse hat damit so wenig Chance, in der gesamten Interessenstruktur Gewicht zu gewinnen, daß sogar noch die Verletzung dieses Interesses zur Realisierung des für Herrn Z. zentralen Interesses, ein guter Arbeiter zu sein, herhalten muß. Dies ist der innere Kern des von Herrn Z. ausgesprochenen Verzichts auf Gesundheit. 
Als Gesundheitsverhalten bezeichnen wir alles Verhalten in der - insbesondere außerberuflichien - alltäglichen Lebenspraxis, von dem die Gesundheit besonders beeinflußt wird, also insbesondere Essen, Trinken, Rauchen, Ruhe, Bewegung, Körperpflege, Gesundheitspraktiken, präventive Gesundheitsüberprüfung u.ä. Es scheint für diese Verhaltensweisen gesellschaftlich (bisweilen auch gruppenspezifisch) geltende Normalwerte zu geben, von denen die Individuen zum großen Teil ihr Handeln leiten lassen. Auch Herr $Z$. orientiert sein Handeln in diesem Bereich an der Folie der Normalität, was ein weiteres Indiz dafür ist, daß sein prekärer Gesundheitszustand von ihm ganz undramatisch erfahren und als Normalschicksal gedeutet wird. Jedenfalls zeigen Essen, Trinken, Rauchen, Ruhe und Bewegung keinerlei extreme Ausschläge. Bestimmte Gesundheitspraktiken, von denen er erzählt - z.B. nimmt er jedes Jahr sechs Moor- oder Schwefelbäder zur Pflege seiner Füße sind zwar nicht allgemein verbreitet, nehmen aber in seinem alltäglichen Leben keinen großen Raum ein. Auf das einzige hier auffallende Faktum, nämlich seine strikte Weigerung, sich einer präventiven Gesundheitskontrolle zu unterziehen, werden wir später noch zurückkommen.

Gerade weil das Gesundheitsverhalten von Herrn Z. weitgehend einer Normalfolie folgt, ist sein Verhalten im Fall von Krankheit um so auffälliger und erklärungsbedürftiger. An den folgenden von ihm berichteten Reaktionen auf das Eintreten von Gesundheitsstörungen wird dies deutlich:

- Herr Z. überspielt, so lange es geht, auch grayierende Krankheitssymptome (z.B. Grippegefühl, hohes Fieber u.ä.) und geht zur Arbeit.

- Bei einer schmerzhaften und zur Bewegungsunfähigkeit führenden Verklemmung eines Rückennervs unternimmt er den (vergeblichen) Versuch, durch ambulante Behandlung im Krankenhaus, das er ohne Umweg über den Hausarzt aufsucht, zu erreichen, daß er sofort wieder arbeitsfähig wird.

- Mit Hilfe von Schmerz- und Grippetabletten, die er regelmäßig vom Werkssanitäter sich beschafft, betäubt er Schmerzen, um seine Arbeit weiterführen zu können.

- Bei einer Krankheitsepisode im Urlaub, den er zum Umziehen nutzen muß, nimmt er nicht die Möglichkeit in Anspruch sich krank schreiben zu lassen, sondern drängt den Arzt zu einer problematischen Symptombehandlung.

- Selbst gefährliche, zum Teil schmerzhafte Veränderungen seines Körpers - z.B. eine große, bị in den Arm hinein verschiebbare Flüssigkeitsblase an der Hand und sogar ein Leistenbruch - können ihn lange Zeit nicht veranlassen, etwas dagegen zu unternehmen.

Man sieht sofort das Gemeinsame an diesen verschiedenen Reaktionsweisen: Herr Z. versucht offensichtlich, eine adäquate Behandlung von Krankheiten und vor allem die im Krankheitsfall erforderliche Ruhe und Schonung seines Körpers zu umgehen, damit es nicht zu einer Unterbrechung seiner Arbeitstätigkeit kommt. Die Motivationsstruktur, die hinter diesem Verhalten steht, läßt sich aus einigen Berichten von Krankheitsepisoden herauslesen:

$B$ : Hab dann weiter gearbeitet, noch drei Wochen gearbeitet und so mit, mit dem Arm, ne, da hab ich das Wasser von do bis her drücken können, von do bis davor drücken können, ne, ne. Ma Meister hat immer gesagt, na geh halt nauf und laß Dir's nachschauen. Sag ich: Na ich geh in Urlaub hab ich 
gesagt, der Arm bleibt nicht mehr so angestrengt, des vergeht. Ja jetzt gingen drei Wochen gingen rum, der Arm ist als stärker geworden. Na do hab ich dann noch 14 Tage gearbeitet nach meinem Urlaub, da sagt mir mei Meister, mei Meister K., da jetzt packst zusammen und gehst nauf, läßt mal nachschauen, des, des gefällt mir auch nicht mehr. Da bin ich dann nauf gegangen zum Sanitätsarzt. $B$ : I tu laufend mit 50 Kilobrocken rumhauen, ne, und, do hat mir's, do hab ich dann a Operation gehabt, das war auch vor drei Jahren gewesen. Das war alles schon so weit gewesen, daß es nicht mehr gegangen ist. Dann, dann die Sani droben gesagt, schau und verschwind, geh zum Arzt. Und dann war ich halt 14 Tage daheim. Und dann wat ich wieder an meiner Arbeit gewesen.

$B$ : Mei Leistenbruch, den hab ich, hab ich drei Jahre so rumgeschleppt, der hat sich gegeben gehabt, da bin ich noch gearbeitet.

I: Mh.

B: Und da hat mir mei Meister so dazu ge.., zugesprochen also, jetzt pack dich zusammen und geh jetzt nun endlich e mal und laß machen, einmal, einmal mußt sowieso am Messer tan, sagt der immer, ne, ne. na ja..

$B$ : ...Weil (?) ich konnt ja nit, nit krank machen, (..) Urlaub nehmen und dann umziehen und dann hergehen und krank machen, und ich, ich, transportier die Möbel do in der Gegend rum und dabei bin ich krank geschrieben, ne. Ich muß ja denken, es kann ja irgendwer ... kommen, und dann bin ich ja plötzlich der Blödèl, ne, also na soweit hab ich schon gedacht, ne also, könnt es vielleicht machen, aber ich hab's trotzdem nicht getan.

Drei Momente sind an diesen Episoden hervorzuheben:

1. Offensichtlich ist der Verzicht von Herrn Z., seine Krankheiten adäquat zu behandeln oder behandeln zu lasșen, nicht Folge eines Drucks, den die Firma - eventuell sogar durch Androhung von Sanktionen - gegenüber kranken Betriebsangehörigen ausübt. Herr Z. nimmt ja nicht einmal entfernt die Möglichkeiten in Anspruch, die die Firma ihren Beschäftigten zugestehen muß. Darüberhinaus hat er offensichtlich aufgrund seines Arbeitseinsatzes und seiner Arbeitsauffassung eine so gesicherte Stellung im Betrieb errungen, daß schon viel zusammenkommen müßte, bevor er Gefahr laufen würde, von der Firma gemaßregelt zu werden. Er könnte durchaus mehr für die Wiederherstellung seiner Gesundheit tun, sicher auch häufiger mal krank feiern, ohne daß er durch Sanktionen von Seiten des Betriebes bedroht wäre. Das beweist nicht zuletzt die Tatsache, daß er von Instanzen des Betriebs (Meister, Sanitäter) sogar dazu aufgefordert wird, sich um seine Krankheiten zu kümmern.

2. Mit diesen Aufforderungen von Seiten der betrieblichen Instanzen, seine Krankheitssymptome ernst zu nehmen und sie nicht zu überspielen, hat es nun eine eigene Bewandnis. Man erkennt dies deutlich, wenn man andere Stellen heranzicht, in denen Herr Z . solche Instanzen erwähnt oder sich auf sie bezieht. Es handelt sich meistens darum, daß diese Instanzen etwas yon ihm 'wissen', 'behaupten können' usw.:

B: ... dann hat mir der Arzt dann mich ne Woche daheim gelassen, ne. Ich hab dann mein Meister glei angerufen. Ha so meinem Kollegen gesagt, der auch am Telefon, er sollt dem Meister Bescheid sagen. Ja, der wußte das ganz genau, wenn ich emal daheim bleib, dann ist schon bestimmt was.

I: Mmh, mmh

B: Das weiß der Herr $\mathrm{G}$. hundertprozentig.

$B$ : Nein, ich hab auch nichts zum Krankmachen gehabt (...) Mein Meister kann dasselbe behaupten, ich hab sechs Jahre durchgehaut ohne, ohne daß ich irgendwie, daß ich gesagt hab, heut mag ich nicht.

$B:$ Ne, ma da könnens könnens mei Meister fragen und auch der vorhergehende Chef, der is schon pensioniert und der heutige ist der Herr Sch., ne, i ma aber, für mich sagen mal, also Eigenlob stinkt heißt's aber ich hab das noch nie getan, daß geheißen hat, na ja hoffentlich vielleicht kummt der heut oder irgendwas, ich war jeden Tag an meiner Arbeit gewesen, ne. 
B: Also i will mich jetzt nicht fei als super dahinstellen und gar nichts aber da können's eigen's, können's persönlich mei, mein Chef, mein Meiste, den Herr X. und mit dem muß ich sprechen, die kennen mich schon seit 25 Jahr', daß ich wart (?) jeden Tag drin gewesen.

Offensichtlich wiederholt sich hier auf einer anderen Ebene das, was Herr Z. als Sich-mitdem-Meister-zusammentun bezeichnet. So wie ganz allgemein sein außerordentlicher Arbeitseinsatz, so muß im besonderen die Aufopferung seiner Gesundheit bei der Erfüllung seiner Arbeitspflicht Anerkennung bei den betrieblichen Instanzen finden. Aber dieser A nerkennung kann man sich nicht ein für allemal sicher sein, sie ist immer gefährdet, und gerade darum muß man sich ihrer durch besondere Anstrengungen immer wieder vergewissern. Schon die Demonstration von schweren Krankheitssymptomen bei der Arbeit erfüllt diesen Zweck sehr wirkungsvoll. A ber eine beinahe hundertprozentige Sicherheit, daß die Anerkennung trotz Fernbleiben aus Krankheitsgründen nicht verloren gcht, erhält man, wenn man die Entscheidung, daß man zu Hause bleiben soll, nicht selber triff $t$, sondern sie durch die betrieblichen Instanzen fällen läßt. Wenn man sich die Absolution ge. ben läßt, bevor man sündigt, kann man sicher sein, daß die Sünde nicht zählt.

Gerade darum fällt es Herm $\mathbb{Z}$. auch so schwer, sich im Urlaub krank schreiben zu lassen. Da ihn hier keine betriebliche Instanz 'freisprechen' kann, wäre es denkbar, daß ein Argwohn sich einschleichen oder Gerüchte sich an sein Krankfeiern heften und damit seinen Ruf schädigen könnten, und darum hält Herr $Z$. es für besser, sich erst gar nicht in diese Gefahrenzone zu begeben.

3. Man darf nun allerdings die permanenten Anstrengungen von Herrn Z., für sein Verhalten Anerkennung bei den betrieblichen Instanzen zu finden, nicht so deuten, als würde er, um den Erwartungen der betrieblichen Instanzen zu genügen, seine eigenen Interessea zurückstellen und seine Anstrengungen nur als Mittel unternehmen, um jene Anerkennung zu erreichen. Dabei würde unterstellt, daß es einen Unterschied gäbe zwischen dem, was Herr Z, von sich aus, und dem, was er zum Zweck der Sicherung seiner Stellung im Betrieb tun.würde. Wir haben oben schon gesehen, daß es für eine solche Differenz keinerlei Anzeichen gibt. Auch hier deutet Herr Z. mit keinem Wort an, daß er von sich aus andere Verhaltensweisen als die, die er vor den Augen der betrieblichen Instanzen ausführe, für sinnvoll halten würde. Es deutet umgekehrt alles darauf hin, daß er die Handlungen, die ihm in den Augen der betrieblichen Instanzen Anerkennung einbringen, selber für vernünftig und erstrebenswert ansieht. Das, was ihm nach außen Anerkennung verleiht, wird von ihm, auch wenn es ein für seine Existenz bedrohliches Moment enthält, als sein eigenes Interesse vertreten.

Nirgendwo zeigt sich deutlicher als ari dieser Stelle, wie ein Subjekt sich in die Dialektik der Unterwerfung verstricken kann, indem es gleichzeitig die Unterwerfung volizieht und sie sich unsichtbar macht. Die Verinnerlichung der betrieblichen Arbeitsverpflichtungen und die dadurch bestimmten Handlungen von Hern $Z$. bringen Prozesse hervor, durch die sich diesem ein auf den Kopf gestelltes Bild des Verhaltnisses von Betriebsinteressen. und Gesundheirsintetessen der Beschäftigten aufdrängen iruß̈. Die besondere Iorm, in der das Arbeitsinteresse von Herrn 2 . in Erscheinung trits, hat zur Konsequenz ein für seine Gssundheit extrem riskantes Verhalten. Gerade dadurch kann der Betrieb ihm gegen. uber die Funktion wahrnehmen, ihn an die Notwendigkeit zi erinnern, für die eigene Gesundheit Sorge zu tragen. Die grundlegende Bestimmung, daß Betriebe für ihre Zwecke die Gesundheit ihrer Beschäftigten in Anspruch nehmen, führt an der Oberfläche zu dem 
entgegengesetzten Resultat: daß der Betrieb das Gesundheitsinteresse gegenüber den unvernünftigen Interessen der Beschäftigten vertritt. Auch von dieser Seite her kann sich daher Herr $Z$. in seiner Ansicht bestätigt sehen, daß die Firma den Gesundheitsinteressen der Beschäftigten nicht entgegenwirkt.

Eingebunden in grundlegende Strukturen seiner Lebenswelt wird das beschriebene Krankheitsverhalten von Herrn Z. mit einer fast unheimlichen Konsequenz durchgehalten. Das zeigt sich gerade auch an seinem Verhältnis zu präventiven, auf die Früherkennung von Krankheiten gerichteten Maßnahmen. Wenn man nicht einmal manifeste Krankheitssymptome zum Anlaß nimmt, möglichst rasch eine Behandlung herbeizuführen, würde es wenig Sinn machen, über die ersten Anzeichen einer Krankheit Kenntnis erlangen zu wollen. Eine Früherkennung, deren Ergebnisse nicht zu praktischen Konsequienzen führen, würde zu eimer bloßen Belastung, weil sie nicht nur Zeit in Anspruch nimmt, sondern oft auch Unruhe und Bcsorgnis auslöst. Es ist darum nichts weiter als eine Radikalisierung seines im Krankheitsfall gezeigten Verhaltens, wenn Herr Z. sich definitiv weigert, die an seinem Körper eintretenden Entwicklungen zur Kenntnis zu nehmen.

$B$ : ... ich sag immer, ich will von da gar nichts wissen, ne, ne. Wenn es jetzt heißt, es heißt, von vielem Kopfweh kriegt man des oder man kriegt das und das, ne, da will ich dann nichts wissen davon. $B$ : Die eine ist, die eine sogt, mir, mir tut mei Herz weh, mir tut do weh, ne, und, und dann jetzt hab ich Verstopfung Beispiel, oder, oder ich hab, ich hab jetzt, ne, des könnt sein oder das könnt sein. Das will ich alles, so was will ich überhaupt nicht wissen.

I: Ja so, daß man einfach das Gefühl hat, also, da könnte sich was tun?

$B$ : Naa, so weit denk ich nicht. So einfach ... wenn, wenn's kummt, wenn was kummt, kummt's ja sowieso en Ausbruch und, und

$B$ : ... Beispiel ich selber will meinen Körper gar nicht kennen, will ich gar nicht kennen...

\section{Fertig Aus Amen.《}

Wir haben gesehen, daß die Gesundheit von Herrn Z. nicht nur durch objektive, ihm unabänderlich vorgegebene Bedingungen bedroht ist, sondern auch durch sein eigenes, von ihm selbst bestimmtes Verhalten. Gerade weil sein eigenes Verhalten, insbesondere die Weigerung, sich präventiv mit seinen Gesundheitsproblemen zu befassen, die Gefahr des Anwachsens dieser Probleme nicht unerheblich erhöht, ist es um so bemerkenswerter, daß Herr Z. in keiner Weise einen Zweifel an der Richtigkeit dieses Verhaltens aufkommen läßt. Von welcher Seite auch immer er auf sein Gesundheitsverhalten zu sprechen kommt, er stellt es so dar, als würde für ihn als Person eine unbedingte Notwendigkeit bestehen, so zu handeln, wie er es tut. Er faßt sein Handeln mit einer so entschiedenen Selbstverständlichkeit auf, daß es gar nicht denkbar wäre, daß er alternative Handlungsmöglichkeiten, Formen einer eher präventiven Behandlung seiner Gesundheitsprobleme, auch nur in Erwägung ziehen, geschweige denn gegen sein eigenes Verhalten abwägen würde. Sein Gesundheitsverhalten hat sich zu einer definitiven Lebensform verfestigt. Wie ist dies möglich?

Zu einem großen Teil erklärt sich die Entschiedenheit, mit der Herr Z. auf der von ihm praktizierten Form des Gesundheitsverhaltens besteht, daraus, daß er dieses Verhalten als notwendig für die Realisierung seines Ideals ansieht, ein guter Arbeiter zu sein und als solcher anerkannt zu werden. Aber dieser Bezug seines Gesundheitsverhaltens zu seinem zentralen Lebensinteresse ist nicht ausreichend. Erstens ist es nicht selbstverständlich, daß ein 
derartig gegen die Gesundheit gerichtetes Verhalten tatsächlich zur Realisierung seines Ideals notwendig ist. Und zweitens würde auch dann, wenn diese Notwendigkeit bestehen sollte, immer noch das Paradox zu bewältigen sein, daß die Realisierung eines sinnvollen Lebens die Zerstörung der Gesundheit einschließen soll.

Verhaltensweisen gewinnen erst dann die unangreifbare Selbstverständlichkeit, wic sie von Herrn Z. demonstriert wird, wenn sie in der Realitätśdeutung des Subjekts, seiner Form der Anschauung und Verarbeitung der Realität, sich niedergeschlagen haben. Die sich verfestigende Lebensform umgibt sich gleichsam mit Anschauungen und Einstellungen, durch die sie 'ideologisch' abgesichert wird. Deren Funktion ist es vor allem die in der Lebensform angelegten Versagungen und Gefahren tragbar zu machen, indem diese teils als solche unkenntlich gemacht, teils unauflöslich mit dem positiven Kern der Lebensform verschweißt werden.

1. Herr Z. könnte seine Lebensform sicherlich nicht mit einem so hohen Grad an Bestimmtheit und Selbstverständlichkeit vertreten, wenn es ihm nicht gelingen würde, die in dieser Lebensform enthaltenen Versagungen und Gefahren einzukapseln und vom Strom des alltäglichen Lebens separiert zu halten. Betrachten wir die von Herrn Z. so betont vorgetragenen Erklärungen, mit denen er seinen Verzicht auf Gesundheit zum Ausdruck bringt, im Zusammenhang mit seiner Weigerung, die Vorgänge an seinem Körper zur Kenntnis zu nehmen, so sehen wir, wie seine Gesundheitsprobleme einem solchen Prozeß der Einkapselung unterzogen werden. Ein Verzicht kann nicht nur Resultat langer und harter Auseinandersetzungen sein, sondern kann auch ausgesprochen werden, um sich solche Auseinandersetzungen zu ersparen. Wenn Herr Z anerkennt, daß er in Zukunft von gravierenden Gesundheitsproblemen betroffen sein wird und dadurch sich sogar seine Lebenszeit verkürzen könnte, dann streckt er gleichsam die Waffen. Wichtig darin ist nicht nur, daß er sich für besiegt erklärt, sondern daß er sich nicht länger in fortdauernde und vielleicht verlustreiche Kämpfe verwickeln lassen muß. Das Schicksal, gegen das man sich sträubt, wirft ständig seine drohenden Schatten auf die Gegenwart. Ergibt man sich jedoch in dieses Schicksal, kann man sich wenigstens für den Augenblick von dessen Last befreien. Das Schicksal anerkennen heißt, sich nicht mehr um es kümmern zu müssen, mit ihm »fertig« zu sein.

B: Genau so wie mir mei Schwester, allmächt na da tu ich eben (?) passen das wird e Thrombose. Sag ich, ich weiß aber gar nicht, was e Thrombose ist, das interessiert mich gar nicht. Wenn das ist, na dann muß man halt zum Doktor gehen und fertig aus Amen, ne.

2. Eine andere Möglichkeit, eine Lebensform vor Zweifeln zu schützen, besteht, darin, den in dieser Lebensform enthaltenen Versagungen und Verletzungen durch Umdeutung und Beschönigung ihren negativen Charakter zu nehmen. Die Tatsache, daß Herr Z. im Prinzip anerkennt, daß er in Zukunft von gravierenden Gesundheitsproblemen betroffeŕ sein wird, hindert ihn nicht daran, im gleichen Augenblick diese Probleme zu verbarmlosen.

B: ... Dann haben die gesagt, also das it mit de Bandscheiben, da hat sich irgendwas gelöst gehabt von der Bandscheibe und ist genau in den Ding neigekommen, das hat die großen Schmerzen gehabt. Die haben mir auch gesagt: So schnell wie die Schmerzen kummen, so schnell werden sie auch vergehen, ne.

$B$ : Na verschlechtert hat er sich halt damit, sagen mer, wo jetzt ab und zu mal die Bandscheiben mal 
so weh tut, ne. Aber so wie's, so wie's kommt, so vergeht's auch: Das hat sich verbe... durch die, verschlechtert durch die Jahre, ja, das stimmt.

I: Der Arzt hat (...) gesagt, daß das mal wieder kommen könnte?

$B$ : Ja, des könnt; das kann schon mal kommen wiedermal. Aber mein Gott, des is dann, in zwei drei Tag ist das vorbei. Also ich hoff jetzt, daß nicht kommt, ne, ich will mir's nicht wünschen, ne.

Herr Z. hat die Vorstellung, daß seine Krankheiten den Charakter besitzen, rasch wieder zu verschwinden, wenn sie auftreten. Das heißt erstens, daß selbst, wenn er krank wird, dies nicht zu länger andauerndem Leiden führt. Oder anders: daß sich mit seinen Krankheiten leben läßt. Und zweitens, daß im Lauf der Zeit an dieser Erscheinungsform seiner Krankheiten sich nichts ändern wird.

Wenn man nicht leugnen kann, daß man sich durch seine berufliche Tätigkeit Gesundheitsprobleme schafft, so kann man wenigstens, was ihr Ausmaß betrifft, eine günstige Prognose stellen. In dieselbe Richtung zielt auch die Relativierung solcher Probleme.

$B$ : Ja, fünf Jahre will ich schon noch machen, ne.

I: Ja.

B: I mein, dann bin ich doch 54,53 , ne. Und dann, ob's mir dann noch so geht, wenn's geht, will ich schon bleiben noch weiter, ne. Aber ich sag, ich will halt, will halt arbeiten. Und wenn's nicht geht, na dann, ja kaputt machen, will ich mich auch wegen dem nicht lassen, ne.

Herr Z. unterscheidet ausdrücklich Arbeiten, das zu Gesundheitsproblemen führt, und Arbeiten, das kaputt macht. Dabei sieht er den letzteren Fall nur dann als gegeben an, wenn die Arbeitskraft schon weitgehend zerstört ist. Wenn aber gilt, daß man sich nur dann durch Arbeit kaputt machen kann, wenn man eigentlich schon nicht mehr arbeiten kann, so gilt umgekehrt, daß man sich nicht durch Arbeit kaputt machen kann, solange man noch arbeiten kann - und das trifft für Herrn Z. zu.

Die Prognose über sein weiteres Schicksal fällt noch günstiger aus, wenn man eine andere Vorstellung von Herrn Z. hinzunimmt, mit der er noch einmal seine Erwartung bestätigt, daß sich auch in Zukunft mit seinen Gesundheitsproblemen leben läßt.

$B$ : Ja daß ich, sage ma ... vielleicht hab ich jetzt Sie falsch verstanden, wenn ich sagen mal, daß ich diese schwere Arbeit sagen mir mit, mit $55 \mathrm{Jahr}$, mit $60 \mathrm{Jahr}$ bestimmt nicht machen kann, des, des des eine weiß ich, das weiß ich. Dann will ich's hoffen, daß mir mein Meister soviel Chancen geben würde, daß er sagt: Gut jetzt mal, du hast jetzt, das ist dann so dreißig, na ja fünfundzwanzig, dreißig Jahr lang ist dauern mit de schwere Ma.., schwere Brocken rumgekämpft. Jetzt kannst de mal was Leichteres auch machen.

3. Inebensformen können ihre innere Sicherheit aus verschiedenen Quellen beziehen. Je mehr aber eine Lebensform durch Versagungen und Verzicht gekennzeichnet ist, um so eher wird sich eine Form der Selbstrechtfertigung einstellen, die auch im größten Unglück noch Halt zu geben vermag: Die Behauptung eines nur der eigenen Existenz zukommenden moralischen Werts. Die Kehrseite einer solchen Selbstrechtfertigung ist gewöhnlich eine fast $z$ wanghafte Neigung, alternative Lebensformen, die sich nicht der Praxis des Verzichts anschließen, moralisch abzuqualifizieren. Für Herrn Z. gibt es einen festen Zusammenhang $z w i s c h e n$ seinem Ideal, ein guter Arbeiter zu sein und der Vernachlässigung von Maßnahmen, die für die Erhaltung der Gesundheit erforderlich wären. Umgekehrt bedeutet dies, daß alle diejenigen, die sich um ihre Gesundheit kümmern - und das heißt für Herrn Z. zuviel um ihre Gesundheit kümmern - keine guten Arbeiter sein können. Daß 
dieser Unterschied von Lebensformen von ihm als ein moralischer Gegensatz, das heißt ein Gegensatz zwischen einer unmoralischen und einer moralischen Lebensform aufgefaßt wird, zeigt sich deutlich genug bei der Charakterisierung der Kollegen, die sich in ihrem Gesundheitsverhalten nicht an das halten, was er für geboten hält.

$B$ : Aber wenn ich heute arbeiten tu und denn anderen Tag tatsächlich, und ich, kaum hab ich, kaum hab ich vier Wochen gearbeitet und da bin ich schon wieder beim Arzt, das ist in meines Achtens noch Berufkrankheit, Berufs-, Berufskrankheit so ne. Die sagen, das wird ja sowieso auch bezahlt, ich krieg ja meinen Durchschnittslohn.

$B$ : ... A ber selber so Krankheit, daß ich sag, so wie's heute ist, ich will keine Namen nit nennen, das will ich nicht machen, aber manche sagen heut; Ach heut gefällt's mir nicht, es ist zu heiß, ich geh zum Arzt, ich bin krank.

I: Oder auch mal umgekehrt, jemand, der besonders gut auf seine Gesundheit achtet?

$B$ : Na ja, da hab ich e Kollege, ja, der gibt Obacht, der sagt, ja nit mehr heben, wie was sein muß, weil (?) sonst ... (lacht) geht's auch nicht mehr so und, und ... besser ausgeruht und, und, und man muß sich mehr schonen. Ich kenn schon solche, ja. Des, des sind lauter solche die was alle, was die Berufskrankheiten, Berufskrankheiten sind, ne. Kenn ich schon solche.

I: Wie schätzen Sie denn die, die ein, die da so vorsichtig sind?

$B: \mathrm{Na}$ ja also, ich schätz sie nicht gut ein. (Unterbrechung) Ma ich schätz die Leute nicht gut ein.

I: Mh. Meinen, sie sind zu vorsichtig oder?

$B$ : Ja, wenn ich, mei ich hab en Kollegen da, was ich kenn, der sagt da: Na ja, ich bin jetzt schon wieder krank. Na da hab ich gesagt, denkt immer: Mensch, bin ich selber blöd. Du raust und rennst und schepperst und, und die hocken daheim und tun ihr Geld genauso verdienen. $\mathrm{Na}$, da hab ich en Brast danti, ne. Aber daß ich, mei ich kann nicht schimpfen mit dem Kollegen wegen dem machst du krank, ne, des, des werd ich nie tun, ne.

Der Begriff der 'Berufskrankheit' ist hier durchaus kritisch gemeint, aber eben nicht in dem Sinn, in dem der Begriff sonst verwendet wird. Daß Krankheiten aus der beruflichen Tätigkeit erwachsen können, weiß Herr Z., aber dies ist für ihn nicht der kritische Punkt. Was er kritisiert, ist, daß Krankheiten oder Anstrengungen zur Sicherung der Gesundheit in den Beruf hineingezogen werden. Der kritisierte Zusammenhang ist nicht der, daß Krankheiten durch die Belastungen des Berufs entstehen, sondern daß Krankheiten zu einer Belastung für den Beruf werden.

Soweit die 'Berufskranken' gar nicht krank sind, also keinen organischen Befund haben, ist ihre moralische Verurteilung nichts Außergewöhnliches. Arbeiter, die Verständnis für Kollegen aufbringen, die ohne krank zu sein krank feiern, finden sich nicht häufig. Für die moralische $A$ bqualifizierung genügt allein das Bewußtsein, daß derjenige, der krank feiert, dieselben Vorteile genießt, wie derjenige, der sich 'abplagt'. Hinzu tritt oft noch die Erfahrung, daß diejenigen, die zur Arbeit gehen, teilweise die Arbeit ihrer krankfeiernden Kollegen mitübernehmen müssen.

Herr Z. aber geht in seiner Kritik noch einen Schritt weiter, indem er den Kreis der 'Berufskranken' auch auf diejenigen ausdehnt, die tatsächlich Gesundheitsprobleme haben, wenn auch nicht so schwerwiegende, daß sie ihre Arbeitsfähigkeit vollständig eingebüßt hätten. Selbst wenn es bei seinen Kollegen »nicht mehr so gut geht", kann Herr Z. sie »nicht gut beurteilen«, wenn sie ihre Arbeitskraft schonend einsetzen. Dieses Urteil über die gesundheitlich schon geschädigten Kollegen ist für Herrn Z. wichtig. Denn durch dieses Urteil rückt er seine eigene Leistung erst ins rechte Licht, die ja gerade darin besteht, trotz gesundheitlicher Probleme mit voller Intensität weiterzuarbeiten und sich nicht um diese Probleme zu kümmern. 
Gleichzeitig ist dieses Urteil aber auch besonders angreifbar. Selbst mit den moralischen Maßstäben betrachtet, auf die sich Herr $Z$. stützt, stellt sich die Frage, ob es wirklich so falsch ist, wenn Kollegen sich aufgrund ihrer angeschlagenen Gesundheit schonen. Umgekehrt wäre an Herrn Z. die Frage zu richten, warum man sich nicht auch dann als ein guter Arbeiter bewähren können sollte, wenn man nicht so rücksichtslos sich gegen die eigene Gesundheit verhält, wie Herr Z. es tut. Haben nicht die Kollegen recht, die ihm »einflöBen« wollen, er solle sich mehr schonen? Es könnte sich hier ein Zweifel einstellen, nämlich der Zweifel, ob der Preis, den Herr Z. für die Realisierung seines Ideals entrichtet, überhaupt gerechtfertigt ist. Es könnte sich dieser Zweifel einstellen, wenn ihm nicht durch einen weiteren 'Zug' von Herrn Z. unwiderruflich der Boden entzogen würde.

4. Der 'Zug', mit dem Herr Z. die letzten Zweifel an der von ihm praktizierten Lebensform ausräumt, besteht darin, dem präventiven Gesunahbitsverbalten seine Zweckmäßigkeit abzusprechen. Wie ist das möglich? Ist es nicht eine Tatsache, daß Heilungschancen sich verbessern, wenn eine Behandlung zu einem möglichst frühen Zeitpunkt nach dem Ausbruch ciner Krankheit cinsetzt? Und läßt sich nicht die Behandlung dadurch vorverlegen, daß der Körper ständig auf das Auftreten verdächtiger Symptome hin beobachtet wird, oder besser noch dadurch, daß Untersuchungen vor dem Auftreten manifester Krankheitssymptome durchgeführt werden?

Nun weist präventives Gesuhdheitsverhalten aber neben dieser zweckmäßigen noch eine andere Seite auf. Nur in den seltensten Fällen haben Krankheiten eine so eindeutige Verlaufsform, daß aus den zuerst auftretenden Symptomen der weitere Entwicklungsgang mit Sicherheit prognostiziert werden kann. In vielen Fällen wird man auf Erscheinungen und Symptome aufmerksam, von denen ganz ungewiß ist, ob sie sich überhaupt zu Krankheiten entwickeln oder entwickeln müssen. Hier gerät das präventive Gesundheitsverhalten leicht in Gefahr, die Orientierung zu verlieren und auf Irrwege zu geraten, die von voreiliger Besorgnis über Hypochondrie bis zur falschen Behandlungsweise reichen. Im schlimmsten Fall trägt das präventive Gesundheitsverhalten durch die Unruhe, die es in den alltäglichen Lebenszusammenhang hineinträgt, und durch seine Tendenz, iatrogenetischen Entwicklungen Vorschub zu leisten, selber zum Entstehen von Krankheiten bei.

Herr Z. legte die Finger gerade auf diese Schwierigkeiten, in die jedes präventive Gesundheitsverhalten geraten kann.

$B$ : Die eine ist, die eine sagt, mir, mir tut mei Herz weh, mir tut do weh, ne, und, und dann jetzt hab ich Verstopfung Beispiel, oder, oder ich hab, ich hab jetzt, ne, des könnt sein oder das könnt sein. Das will ich alles, so was will ich überhaupt nicht wissen.

I: Mh. Sie meinen also, wenn mian da sich zu viel vorstellt ...

$B$ : Genau, dadurch kommt die Krankheit.

I: $\mathrm{Mh}, \mathrm{mh}$. Aber ich meine ...

B: Genau so wie mir mei Schwester, allmächt na da tu ich eben (?) passen, das wird e Thrombose. Sag ich, ich weiß aber gar nicht, was e Thrombose ist, das interessiert mich gar nicht. Wenn das ist, na dann muß man halt zum Doktor gehen und fertig aus amen, ne. Und da sind, da sind sehr viele Krankheiten, was von meines Erachtens nach als Einbildung kommt oder itgendwie.

I: Mh, mh. Na ja, insofern gibt's ja doch schon Krankheiten, die nicht nur Einbildung sind, ne?

$B$ : Ja mei, des gibt's schon, aber ich sag immer, ich will von da gar nichts wissen, ne, ne. Wenn es jetzt heißt, es heißt, von vielen Kopfweh kriegt man des oder man kriegt das und das, ne, da will ich dann nichts wissen davon. Ich sag immer, daß, daß, wenn man, wenn man sich einbildet: Allmächt da tut mir des weh, das könnt ja so ausarten, dann wir, bild' man sich ja so viel ein, daß man tatsächlich sagt, 
jetzt ist man krank.

I: Ja. Mh. Åh, Sie haben so insgesamt Glück gehabt, so mit Ihrer Gesundheit. Worauf würden Sie denn das zurückführen?

$B: \mathrm{Ne}$, also, zurückführen? So für meine Geschwister, wir waren zu dritt gewesen. Meine Schwester ist schon mit 29 Jahren mit Herz, da die immer schon e schlecht Herz gehabt hat, (...) schon gestorben. Mei andere Schwester, die, die mit, die hat jetzt mit dem Wasser zu tun, nicht, und der einzige, der was eigen ... des ist, ich sag immer wieder, des ganze Dinger, das ist eine Einbildung und viele Krankheit kommt bloß von die, von der Einbildung, Beispiel ich selber will meinen Körper gar nicht kennen, will ich gar nicht kennen, ich sag ...

Statt sich auf das Problem einzulassen, wie die zweckmäßigen Formen des präventiven Gesundheitsverhaltens getrennt von seinen Auswucherungen praktiziert werden können, erklärt er derartige Irrwege für die eigentliche Natur der Prävention und verleiht ihnen dadurch ein solches Gewicht, daß die zweckmäßigen Formen des präventiven Verhaltens dahinter ganz verschwinden. In letzter Konsequenz führt diese Umdeutung zu der Vorstellung, daß das präventive Sich-kümmern um die eigenen Gesundheitsprobleme als eine der Hauptursachen von Krankheit zu gelten hat.

Damit ist nun erstens der extensive Gebrauch der Kategorie 'Berufskrankheit' gerechtfertigt. Es ist im Grunde nicht entscheidend, ob jemand bereits mit einem bestimmten Krankheitsbefund oder ohne einen Solchen sich um seine Gesundheit kümmert, denn es kann unterstellt werden, daß ein vorhandener Krankheitsbefund sich erst als Folge dieses Gesundheitsverhaltens eingestellt hat. Die Berufskranken verwandeln sich von Opfern gesundheitsgefährdender Umstände in solche, die ihre Krankheit selber herbeigeführt haben. Zweitens ist nun jeder Zweifel an dem von Herrn Z. praktizierten Verhalten gegenüber seinen Gesundheitsproblemen ausgeräumt. Nicht nur dient dieses Verhalten der Durchsetzung seines zentralen Lebensinteresses, nicht nur begründet es moralische Dignität, sondern es hilft letztlich sogar, die Gesundheit zu erhalten. Die Vermeidung der Erkenntnis und Behandlung von Krankheit ist zui Vermeidung der Krankheit selber geworden. Die Rücksichtslosigkeit gegenüber der eigenen Gesundheir schlägt in der Vorstellung von Herrn Z. am Ende in ihr Gegenteil um: die Bewahrung der Gesundheit.

\section{Die Politik der Moral}

Unter den Erfahrungen der Beschäftigten, auf die gewerkschaftliche Strategien zur Gesundheitssichẹrung im Betrieb sich gerne stützen, sind zwei von besonderer Bedeutung:

- die Erfahrung, daß die eigene Gesundheit schon angegriffen oder doch in hohem Ausmaß gefährdet ist, und

- die Erfahrung, daß die schon vorhandenen oder noch drohenden Gesundheitsprobleme wesentlich durch die betrieblichen Arbeitsbedingungen mitverursacht sind.

Am Beispiel von Herrn $\mathbb{Z}$. können wir ablesen, daß̧ diese Erfahtungen keineswegs »für sich« sprechen, es vielmehr davon abhängt, in weichem größeren Kontext, letztlich: in welchem lebensweltlichen Zusammenhang sie auftreten.

Für Herrn $Z$. folgt aus diesen Erfahrungen mit Sicherheit nicht die Konsequenz, daß eine gegen den Betrieb gerichtete Strategie der Gesundheitssicherung erforderlich sei. Vor allem würde Herr Z. nicht verstehen, wieso eine Strategie, die sich vom Interesse der Beschäftigten leiten läßt, in einen Gegensatz zum Betriebsinteresse geraten sollte. Denn der 
Betrieb tut seinerseits, wie Herr Z . bemerkt, viel für die Gesundheit der Beschäftigten. Eine vernünftige Strategie der Gesundheitssicherung - hätte also weiter nichts zu tun, als die vom Betrieb bereitgestellten Möglichkeiten zu nutzen.

Es versteht sich von selbst, daß eine Politik, die nur offene Türen einrennt, keine Sache des entschiedenen Forderns und des kämpferischen Einsatzes ist. Wenn Herr Z deshalb energisch für notwendig herbeizuführende Änderungen plädiert, hat er etwas ganz anderes im Sinn als die Sicherung der Gesundheit im Betrieb. Wir wissen schon, von welcher Seite aus Herr Z. das gemeinsame Wohl von Betrieb und Beschäftigten gefährdet sieht. Der praktische Schluß, den Herr Z. aus dieser seiner Auffassung zieht, ist nur konsequent:

B: ... Und ich würd auch das mehr vorschlagen, das war sehr gut gewesen wie damals noch die Trauensärzte waren ...

1: Vertrauensärzte

B: Ne, da hat's halt geheißen, gut jetzt nach drei Wochen, nach drei Tage hat man en Schein gekriegt, da mußt man danein, ich war persönlich noch nicht gewesen, aber ich wußt (ich hör's?) für die anderen, ne, und es hat's dann, und da ist dann geheißen, du mußt gehen ne, oder man ist nicht krank.

I: $\mathrm{J}_{2}$

B: Und heut, wenn ich zum Arzt geh, ich weiß das halt ganz genau, wern ich heute zum Arzt geh und sag: İch bin krank, mir tut das und das weh, der schreibt mir auf, sagt der: Na gut ist, da bleiben se daheim emal; aber ich werd es nie tun.

Und um den 'Berufskranken' auch noch die letzte Möglichkeit zu nehmen, durch die Maschen dieses Kontrollnetzes zu schlüpfen, werden die Kontrollen noch um eine weitere Stufe ausgebaut.

$B$ : Ich würde auch sagen, wir haben sehr viele, ne, meines Exachtens, meiner Anschauung tach würde ich sogar dem Arzt die Lizenz entziehen, ne, also den Beruf entziehein, denn die sind nicht krank.

Eine Politik, die die Moral der Subjekte verbessern will und gleichzeitig die reellen Grundlagen ihres 'unmoralischen' Handelns ausblendet, kann moralische Zustände nur erzwingen. Die extremen Konsequenzen, zu denen in unsetem Beispiel eine solche Politik getrieben wird, sollten nicht darüber hinwegtäuschen, daß der Standpunkt dieser Politik nicht nur außerhalb der Gewerkschaften und dann of gegen sie bezogen wird, sondern in vie?fältiger Abstufung und Modifkationen die Gewerkschaftsbewegung selber durchzieht und gleichsam von innen heraus eine auf der Aralyse der reellen Lebensverhältnisse gegründete Politik der Interessenvertretung blockiert. 